\title{
Internal Flow Structure of Torque Converter Analyzed with Dynamic Mode Decomposition under Steady Driving Condition
}

\author{
Yasunori Kunisaki ${ }^{1)}$, Shigeru Murata ${ }^{2)}$, Yohsuke Tanaka ${ }^{2)}$ \\ 1) Graduate School of Engineering, Kyoto Institute of Technology, Matsugasaki, Sakyo-ku, Kyoto 606-8585, Japan \\ EXEDY Corporation, 1-1-1, Kidamotomiya, Neyagawa-shi, Osaka, 572-8570, Japan (E-mail: y-kunisaki@exedy.com) \\ 2) Faculty of Mechanical Engineering, Kyoto Institute of Technology, Matsugasaki, Sakyo-ku, Kyoto 606-8585, Japan
}

Received on June 6, 2020

\begin{abstract}
The structure of the flow field inside a torque converter was quantitatively evaluated using dynamic mode decomposition (DMD). The input time series data in the interference region of the cascade blades were measured using particle image velocimetry (PIV). The spatiotemporal flow field was decomposed by DMD into several modes. Each DMD mode corresponds to the frequency of the flow field fluctuation. The flow field frequency mainly consisted of blade passing frequencies and their harmonics. The flow field was visualized for each DMD mode. Positive and negative vortex structures were generated alternately in the direction of rotation of the impeller and turbine. It was found that the vortex structure became smaller at a lower speed ratio.
\end{abstract}

KEY WORDS: power transmission, automatic transmission, measurement / torque converter, PIV, DMD [A2]

\section{Introduction}

The automotive torque converter is one of the most important components in an automatic transmission. The torque converter consists of three elements: an impeller, a turbine, and a stator. The convertor has several basic functions. The function of creep starting and continuous speed variation provides a smooth take-off and better acceleration performance. The damping function reduces the engine torque fluctuation, which reduces noise and vibration of the drive train. Because the transmission efficiency of an oil-driven torque converter is less than that of a mechanical clutch system, the fuel consumption efficiency of vehicles that have an automatic transmission system is lower than that of vehicles having a manual transmission.

Recently, the need to reduce fuel consumption and increase the acceleration performance of vehicles is increasing from the viewpoint of energy consumption and global environmental problems. It is well known that the hydrodynamic performance of the torque converter significantly affects the performance of vehicles. Further, the demand to reduce the axial dimensions of the powertrain is rapidly increasing, and changes such as redesigning the shape of the torque converter to be elliptical and enlarging spaces for the lock-up clutch and the damper are being considered. To satisfy these demands, it is necessary to optimize the shape of the blades and the flow passages of the torque converter. To attain this goal of an optimal design, it is necessary to understand the details of the fluid behavior in the flow passages.

The internal flow field in a torque converter is an unsteady flow field where blades in the cascade array interfere with each other. It is well known that for a flow field in a torque converter composed of a finite number of blades, the blade passing frequency, which depends on the number of blades and the rotational speed of each cascade, has a remarkable influence on the flow characteristics. The working fluid (oil) in the torque converter circulates through each cascade. Therefore, several frequencies overlap each other in the circulation process, and the frequency characteristics of the flow field become complicated. Furthermore, the frequency characteristics depend on the rotational speed of each cascade according to the driving conditions for the vehicle. To properly resolve problems such as noise and vibration and satisfy performance demands, it is necessary to understand the characteristics of these frequencies in detail.

Computational fluid dynamics (CFD) has been widely used to understand the internal flow structure of torque converters. Significant analysis results for flow field characteristics, such as separated and secondary flows, have been reported. ${ }^{(1-4)}$ With the assistance of more advanced computers, large eddy simulations have been applied to the internal flow in a torque converter, and the results indicated that it is necessary to perform unsteady calculations to understand the internal flow field. ${ }^{(5)}$ However, CFD still has limitations, and must be validated by actual experimental results. An experimental study using laser Doppler velocimetry showed that the flow field changes at the relative position of the cascades with respect to each other. ${ }^{(6)}$ Another case study reported that the phase-averaged velocity maps according to the relative angles of the dynamic to the static cascades were reconstructed to visualize characteristics of this unsteady flow field. ${ }^{(7)}$ There has also been a report on the effect of the stator on the frequency characteristics by applying a wavelet transform to the flow field around the stator. ${ }^{(8)}$

As described above, the internal flow field in the torque converter has been clarified by numerical and experimental analyses, and a lot of information has been obtained. However, because the information acquired from these analyses is so large, it is very difficult to determine which design parameters for the torque converter, such as blade shape, are effective for performance improvement. Therefore, it is necessary to 


\section{Yasunori Kunisaki et al / International Journal of Automotive Engineering}

Vol.11, No.3(2020)

analytically organize the spatiotemporal data obtained by fluid analysis, and to investigate a method for extracting the structure of a few unknown latent features.

Recently, there have been many reports on dynamic mode decomposition (DMD) as a multi-dimensional time series analysis technique. ${ }^{(9-14)}$ DMD has been proposed in the field of fluid analysis as a method for extracting feature structures from spatiotemporal data obtained by experimental and numerical analysis. DMD is different from proper orthogonal decomposition (POD), which is used for similar purposes. A feature of DMD is that it is possible to obtain both spatial and dynamic structures simultaneously.

The objective of the present study was to quantitatively evaluate the flow field structure in the torque converter by applying DMD to the time-series flow data obtained from measurements using particle image velocimetry (PIV).

\section{Plexiglass torque converter and experimental apparatus}

\subsection{Plexiglass torque converter for visualization}

A 3D-CAD model of the torque converter and the measurement region for visualization are shown in Fig. 1. This is one of our products, a standard elliptical torque converter. The fundamental torque converter performance is as follows: the stall torque ratio is 1.7 and the speed ratio of coupling point is 0.75 . The torus consists of an impeller, a turbine, and a stator, which make up the fluid coupling part of the torque converter. The outer diameter of the torus and the flatness ratio are $241 \mathrm{~mm}$ and 0.55 , respectively. The number of blades in the impeller, turbine, and stator is 41,37 , and 35 , respectively. Some torque converter dimensions, such as blade thickness, were partly modified in order that the plexiglass torque converter is not fractured. Each part was machined under numerical control from a block of colorless transparent Plexiglass, and they were then assembled together. To minimize the influences of refraction and scattering of the laser sheet, oil with a refractive index of 1.5 , which is almost the same as that of the Plexiglass (1.49), was used. During operation, the temperature of the oil was carefully controlled at $35{ }^{\circ} \mathrm{C} \pm 2{ }^{\circ} \mathrm{C}$. Also, to minimize the effects of reflection from the curved wall, the entire torque converter was submerged in a plexiglass box filled with the same oil, as shown in Fig. 1.
The input shaft rotational speed during measurement was $200 \mathrm{rpm}$. The flow fields were measured at speed ratios of $0.1,0.4$, and 0.7 to measure flow properties under the conditions of stall, acceleration, and cruising, respectively. All measurements were made under steady-state driving conditions. The representative Reynolds number was estimated to be $6 \times 10^{4}$ using the geometrically averaged radius of the flow passage, the mean flow velocity of the impeller outlet calculated from one-dimensional analysis, and the kinetic viscosity of the working fluid.

\subsection{PIV measurement system}

A schematic of the experimental setup is shown in Fig. 2. It consists of the transparent torque converter for visualization, a mechanism to hold and drive the torque converter, and a hydraulic circuit for circulating the working fluid. The mechanical part consists of an input motor used to drive the converter impeller, and an output motor used to absorb the turbine power. Torque from the input and output motors is transmitted to the input and output shafts of the torque converter through timing belts. The angular positions of the impeller and turbine are detected by encoders attached to the input and output shafts, respectively. The hydraulic circuit consists of a hydraulic pump, a tank, several pipes, and the torque converter. The working fluid flows into and out of the torque converter via the shaft, as in an actual vehicle hydraulic circuit. Temperature sensors are attached to the inlet and outlet pipes of the hydraulic circuit. The temperature of the working fluid is controlled by a heater and an air cooling system, allowing measurement under constant temperature.

The control equipment consists of a control panel, a function generator (Iwatsu Electric Co., Ltd., SG-4115, 1,000 Hz), and a data logger (Graphtec Co., midi LOGGER HV GL2000). The control panel controls the rotational speed and working fluid pressure, and processes the angle signals transmitted from the encoders on the input and output shafts. The function generator sends a TTL trigger signal to synchronize each device, and controls the recording timing of the high-speed camera. The data logger records the trigger and angle signals.

The optical equipment for capturing the particle images consists of a continuous emission laser (Viasho, VA-II-N-532; power, $3 \mathrm{~W}$; wavelength, $532 \mathrm{~nm}$; laser light sheet thickness, about $2.0 \mathrm{~mm}$ ) and a high-speed camera (Photron Ltd.,

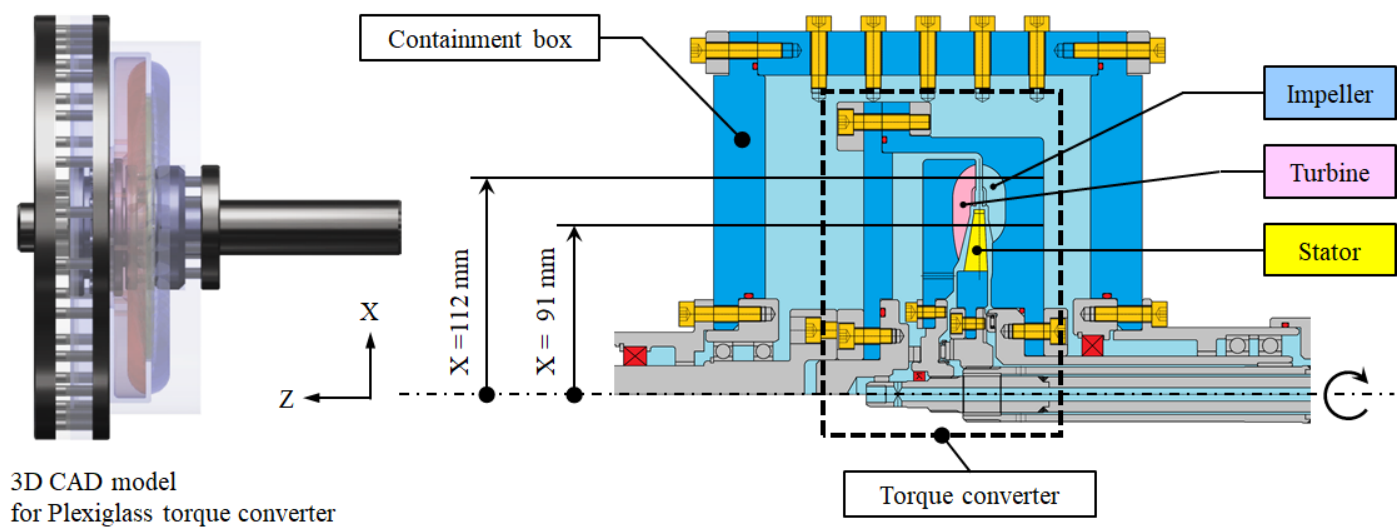

Fig. 1 Plexiglass torque converter and cross section of measurement region. 


\section{Yasunori Kunisaki et al / International Journal of Automotive Engineering}

Vol.11, No.3(2020)

FASTCAM mini UX100, 1,280×800 pixels, 8-bit dynamic range, time interval $0.125 \mathrm{~ms}$ ). The continuous-wave laser is used to supply the laser light sheet to illuminate the flow field. The laser light sheet is reflected by the mirror to illuminate the measurement region. The camera is used to capture the particle images. The tracer particles used for visualization are made from nylon 12, and have an average diameter of $50 \pm 0.2 \mu \mathrm{m}$ and a specific gravity of 1.03 . The working fluid is silicone oil with a specific gravity of 0.995 and a kinematic viscosity coefficient of $15 \mathrm{~mm}^{2} / \mathrm{s}$. The tracer particles and oil have nearly the same specific gravity in order to improve the tracing ability. The resolution of the images obtained in this experiment was 0.05 $\mathrm{mm} /$ pixel. The obtained PIV images were divided into $32 \times 32$ pixel interrogation regions. In this case, the spatial resolution corresponds to $1.6 \times 1.6 \mathrm{~mm}$. The average number of tracer particles appearing in each interrogation region was about 10.

The direct cross-correlation method, ${ }^{(15)}$ which is commonly used in PIV studies on fluid mechanics, was used to calculate the velocity vectors. Also, a correlation-based correction technique, (16) in which the peak value of the cross-correlation coefficient is high and improvement in the signal-to-noise ratio is expected, was used in the image processing algorithm. Sub-pixel analysis using a Gaussian distribution was employed to obtain the velocity vectors with a measurement accuracy of smaller than one pixel, and a statistical method was adopted to eliminate erroneous vectors. Finally, the erroneous vectors were processed by distinguishing the fluid from the physical structure by using image masks corresponding to the rotational angular position of the blades.

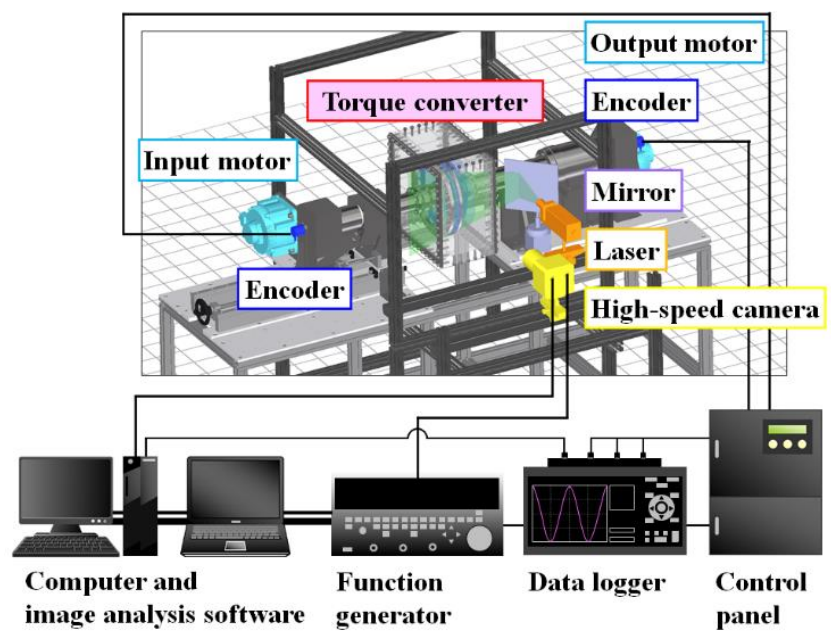

Fig. 2 Schematic of experimental setup.

The flow field was measured in two cross sections. One was at the inner circumference $(X=91 \mathrm{~mm})$ of the torus, at the location of the stator cascade, and the other was at the outer circumference $(X=112 \mathrm{~mm})$ of the torus. A set of 5,120 particle images were captured over $5.12 \mathrm{~s}$ for each cross section.

\subsection{Fundamental blade passing frequency}

The fundamental blade passing frequency for the internal flow field of the torque converter is characterized by the number of blades and the rotational speed. Table 1 shows the frequencies for the impeller and turbine for the three speed ratios employed in this study. Here, the fundamental blade passing frequencies of the impeller and turbine are denoted by $f_{I}$ and $f_{T}$, respectively.

Table 1 Fundamental blade passing frequency for each cascade at input rotational speed of $200 \mathrm{rpm}$.

\begin{tabular}{lrcc}
\hline & \multicolumn{3}{c}{ Speed ratio [ - ] } \\
\cline { 2 - 4 } & 0.1 & 0.4 & 0.7 \\
\hline Impeller $: f_{I}$ & 136.7 & 136.7 & 136.7 \\
Turbine $: f_{T}$ & 12.3 & 49.3 & 86.3 \\
\hline & & & Units [ Hz ]
\end{tabular}

The distributions of velocity vectors and vorticity contours for a speed ratio of 0.1 at $X=91 \mathrm{~mm}$ and $X=112 \mathrm{~mm}$ are shown in Figs. 3(a-c) and 3(d-f), respectively. Figs. 3(a) and 3(d) show the mean flow ensemble. Time series results for each cross section are shown in Figs. 3(b,c) and Figs. 3(e,f) to investigate the change of the flow structure. The time interval is the time during which the upstream cascade moves a distance of $1 / 2$ pitch, or half the blade interval. For the cross section at $X=91 \mathrm{~mm}$ in Figs. 3(ac), the flow direction is from the turbine to the impeller (from left to right). That is, the turbine is the upstream cascade. For the cross section at $X=112 \mathrm{~mm}$ in Figs. 3(d-f), the flow direction is from the impeller to the turbine (from right to left). That is, the impeller is the upstream cascade. The velocity vectors are shown in a stationary coordinate system for the cross section at $X=91$ $\mathrm{mm}$ and in a rotational coordinate system for the cross section at $X=112 \mathrm{~mm}$. The color map shows the vorticity. The structure of the instantaneous field shown in Figs. 3(b,c) is also different from that of the mean flow field shown in Fig. 3(a). In Figs. 3(b,c), in the first time interval, the main flow passes along the suction side of the stator in the center of the figure. In the second interval, the flow collides with the pressure side of the stator and generates a clockwise circulation. The structure of the flow field changes according to the phase of the cascades. In Figs. 3(d-f), a tip vortex is generated at the turbine blade edge and flows into the turbine passage. Clockwise circulation is generated on the pressure side of the turbine blades. It is considered that this circulation is generated due to the difference between the turbine blade inlet angle and the inflow angle of the working fluid from the impeller. These results show that the structure of the flow field is changed by flow interactions between the cascade blades of the impeller, turbine, and stator. For a speed ratio of 0.1 , the changes in the flow structure are larger for the cross section at $X=91 \mathrm{~mm}$ than for the cross section at $X=112 \mathrm{~mm}$.

\subsection{FFT analysis}

Section 2.3 showed that the flow field structure changes dynamically. Therefore, a fast Fourier transform (FFT) analysis for the time series results for measured vorticity was performed to evaluate the frequency characteristics of the internal flow field. The analysis points $\mathrm{P}_{\mathrm{A}}, \mathrm{P}_{\mathrm{B}}$, and $\mathrm{P}_{\mathrm{C}}$ shown in Figs. 4(a,d) are set in the region of unsteady flow due to the flow interactions between the cascade blades of the impeller, turbine, and stator. The time series results for the vorticity at analysis point $\mathrm{P}_{\mathrm{A}}$ for speed ratios of 0.1, 0.4, and 0.7 are shown in Fig. 4(b). Power spectra obtained 


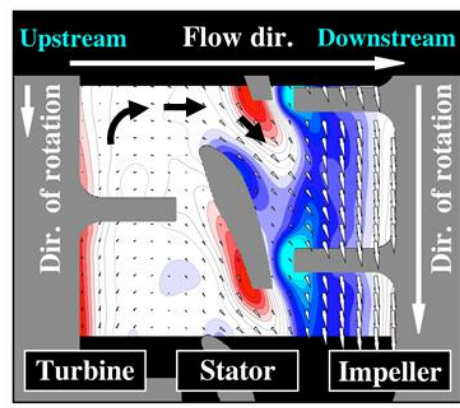

(a) Mean flow

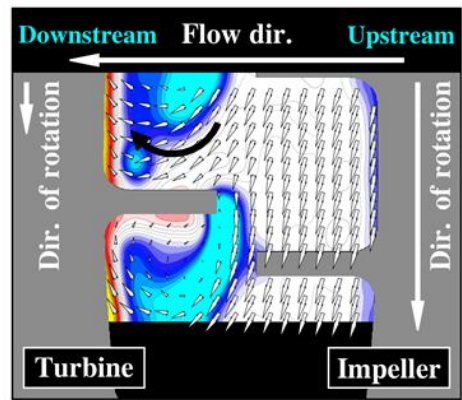

(d) Mean flow

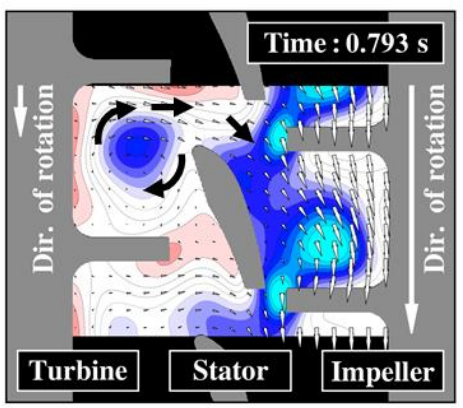

(b) Time interval: $1 /\left(2 f_{\mathrm{T}}\right)$

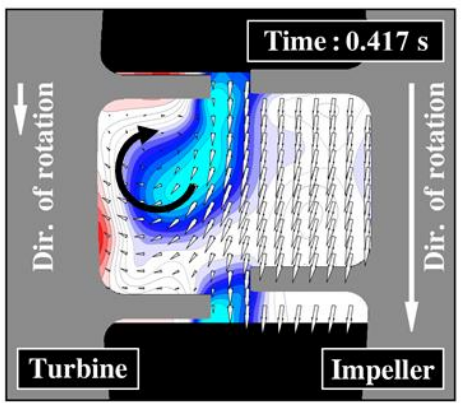

(e) Time interval: $1 /\left(2 f_{\mathrm{I}}\right)$

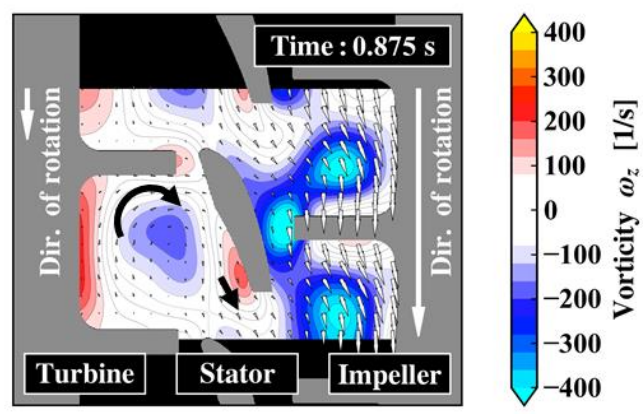

(c) Time interval: $2 /\left(2 f_{\mathrm{T}}\right)$

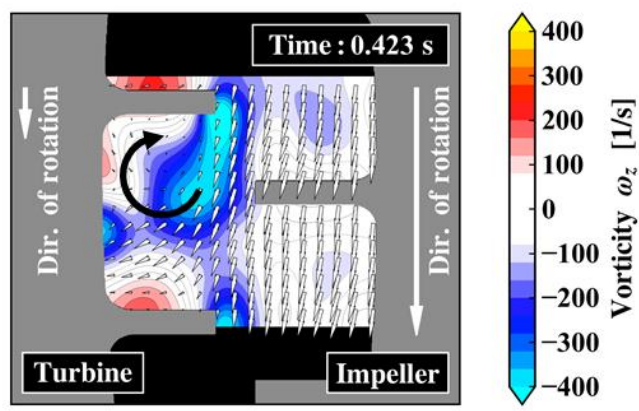

(f) Time interval: $2 /\left(2 f_{\mathrm{I}}\right)$

Fig. 3 Distributions of velocity vectors and vorticity contours for speed ratio of 0.1 .

(a) (b) (c) cross section at $X=91 \mathrm{~mm}$, (d) (e) (f) cross section at $X=112 \mathrm{~mm}$.

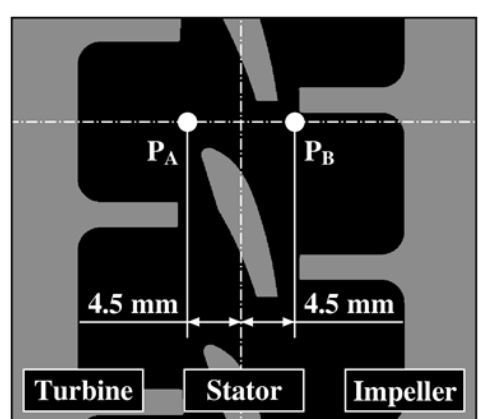

(a) Analysis points at $X=91 \mathrm{~mm}$

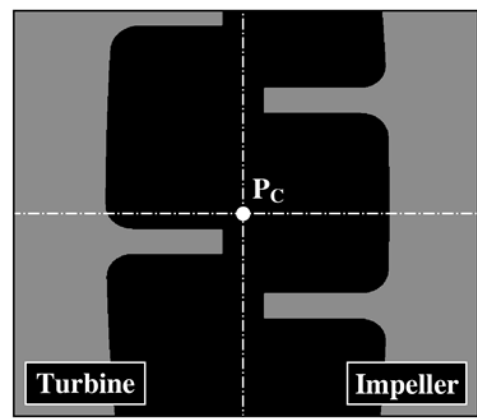

(d) Analysis point at $X=112 \mathrm{~mm}$

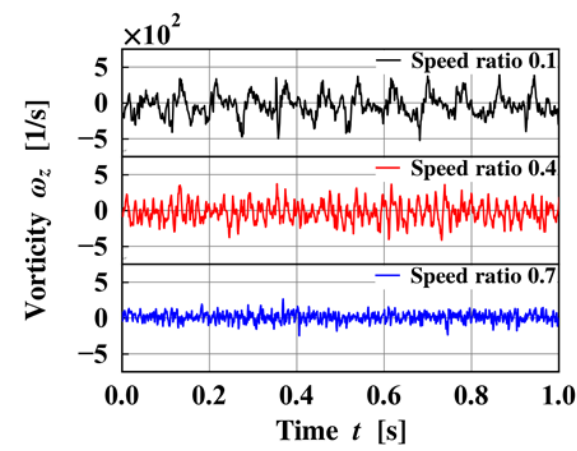

(b) Time series of vorticity

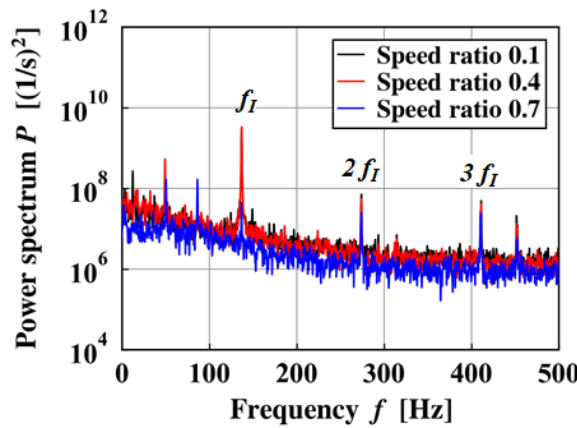

(e) FFT results at $\mathrm{P}_{\mathrm{B}}$

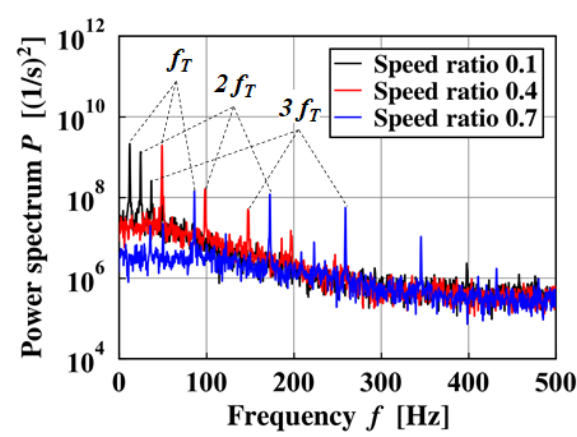

(c) FFT results at $\mathrm{P}_{\mathrm{A}}$

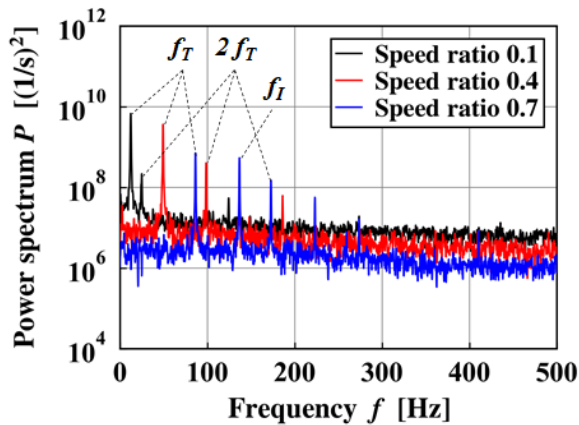

(f) FFT results at $\mathrm{P}_{\mathrm{C}}$

Fig. 4 Positions of analysis points, time series of vorticity, and FFT results for speed ratios of 0.1, 0.4, and 0.7. 


\section{Yasunori Kunisaki et al / International Journal of Automotive Engineering}

Vol.11, No.3(2020)

from the FFT analysis for speed ratios $0.1,0.4$, and 0.7 are shown in Figs. 4(c), 4(e) and 4(f) respectively. At each analysis point, spectral peaks can be found at the blade passing frequencies in Table 1 and harmonics according to the speed ratios.

\section{DMD applied to PIV results}

\subsection{Dynamic mode decomposition}

The results of FFT analysis revealed that the internal flow fields in the torque converter were complicated, with many frequencies superimposed. Therefore, DMD ${ }^{(10-12)}$ was applied to investigate the structure of these flow fields. DMD is a method for decomposing time series data for a flow field into several modes, and extracting the spatiotemporal feature structure. Recently it has been widely used in numerical simulations and physical experiments.

Assume that the flow field data satisfy the following linear system:

$$
x_{i}=A x_{i-1}, i=1,2, \cdots, N,
$$

where $x_{i}$ is the input vector for the $i$-th time step and $N$ is the number of time steps. In this study, the input vector $x_{i}$ is composed of flow velocity vectors. The feature structure is obtained as eigenvalues and eigenvectors of the linear mapping matrix $A$.

The linear approximation is as follows:

$$
Y=A X
$$

where the matrices $X$ and $Y$ are defined by

$$
\begin{aligned}
& X \triangleq\left[\begin{array}{llll}
x_{0} & x_{1} & \cdots & x_{N-1}
\end{array}\right] \\
& Y \triangleq\left[x_{1} x_{2} \cdots x_{N}\right] \text {. }
\end{aligned}
$$

The practical approximation of $A$ is represented by

$$
\tilde{A} \triangleq U^{*} Y V \Sigma^{-1}
$$

where $U, \Sigma$, and $V$ are given by the singular-value decomposition of $X$ :

$$
X=U \Sigma V^{*},
$$

with $(*)$ indicating that the operator is Hermitian. Solving the eigenvalue problem for $\tilde{A}$,

$$
\tilde{A} W=\lambda W
$$

provides eigenvalues $\lambda$, eigenvectors $W$, and the corresponding DMD modes $\Phi$ :

$$
\Phi=Y V \Sigma^{-1} W .
$$

The growth rate and frequency of DMD modes are computed by

$$
\begin{aligned}
& f=\frac{\operatorname{Re}(\log \lambda)}{2 \pi \Delta t}, \\
& g=\frac{\operatorname{Im}(\log \lambda)}{\Delta t},
\end{aligned}
$$

where $\operatorname{Re}(\cdot)$ and $\operatorname{Im}(\cdot)$ denote the real and imaginary parts of a complex value.

\subsection{DMD results}

The eigenvalues contain information about the dynamic behavior of the corresponding DMD modes. The eigenvalues have a real part and an imaginary part. The real part is transformed into frequency $f$ by Eq. (9), and the imaginary part is transformed into growth rate $g$ by Eq. (10).

Distributions of the eigenvalues on complex planes are shown in Fig. 5. Figs. 5(a-c) shows the eigenvalues for a speed ratio of 0.1 for the cross section at $X=91 \mathrm{~mm}$, for a speed ratio of
0.1 for the cross section at $X=112 \mathrm{~mm}$, and for a speed ratio of 0.7 for the cross section at $X=91 \mathrm{~mm}$, respectively. When the eigenvalue is inside the unit circle, the mode is attenuated. When the eigenvalue is outside, the mode is amplified. Eigenvalues lying on the unit circle indicate a steady mode that is not amplified or attenuated. Here, when the absolute value of the eigenvalue is between 0.9 and 1.1, the DMD mode is assumed to be steady. This range is shown in light blue in Fig. 5. It is found that the steady mode is less prevalent for the speed ratio of 0.1 for the cross section at $X=112 \mathrm{~mm}$ in Fig. 5(c) than for speed ratios of 0.1 and 0.7 for the cross section at $X=91 \mathrm{~mm}$ in Figs. 5(a,b). This means that the flow field for the cross section at $X=91 \mathrm{~mm}$ is more complicated than that at $X=112 \mathrm{~mm}$.

The Euclidean norm of the DMD modes $\|\Phi\|$ is used for sorting them, together with the associated frequencies, which is useful for identifying the modes associated with the most physically relevant phenomena. ${ }^{(14)}$ The results of calculating the magnitude of the DMD mode at the cross sections at $X=91 \mathrm{~mm}$ and $X=112 \mathrm{~mm}$ are shown in Figs. 6(a-c) and 6(d-f), respectively. The higher $\|\Phi\|$ values are found remarkably at the blade passing frequencies and the 2nd and 3rd harmonics of the impeller and turbine. The frequencies are consistent with the results of the FFT analysis in Section 2.4 and Figs. 4(c), 4(e) and 4(f). Therefore, the results of the DMD analysis in this study are considered to be reliable.

Figs. 7 (a,b) show the distributions of vorticity contours and velocity vectors corresponding to each DMD mode for the cross section at $X=91 \mathrm{~mm}$ for a speed ratio of 0.1 . The visualized vorticity distributions that characterize the structure of the flow field for the cross sections at $X=91 \mathrm{~mm}$ and $X=112 \mathrm{~mm}$ are shown in Figs. 8(a) and 8(b), respectively. The orange dotted lines in the figures indicates the position of the blade edge. In Fig. 8(a), it can be seen that positive and negative vortex structures are generated alternately in the direction of rotation of both the impeller and turbine. In addition, the repetition spatial frequency of the vortex structure corresponds to the blade pitch of the impeller and turbine. The vortex structure is subdivided in the direction of rotation at the 2 nd harmonics. A similar subdivision of the vortex structure can also be found for speed ratios of 0.4 and 0.7. This is also seen for the cross section at $X=112 \mathrm{~mm}$ in Fig. 8(b). Regarding the flow direction, it is found that the vorticity distribution of the 2 nd harmonics has a three-layer structure along the axis of rotation for a speed ratio of 0.1 . However, as shown within the pink dashed boxes in Fig. 8(b), the subdivided vortex structure merges as the speed ratio increases to 0.4 and 0.7. It is considered that shear flow at the blade edge affects vortex subdivision. The shear flow is caused by the difference in rotational speed between the impeller and turbine. Because this difference becomes larger at lower speed ratios, the vortex structure is considered to be more finely subdivided for a speed ratio of 0.1 than at speed ratios of 0.4 and 0.7 .

\section{Conclusions}

In this study, the unsteady flow field created by flow interactions between cascade blades was visualized, and the velocity field was measured using PIV. DMD was applied to the 
time series flow data obtained by PIV, allowing the structure of the flow field in the torque converter to be quantitatively evaluated. The results obtained in this study can be summarized as follows:

(1) It was found that the structure of the flow field is changed by flow interactions between the cascade blades of the impeller, turbine, and stator. At a speed ratio of 0.1 , the changes in the flow structure were larger for the cross section at $X=91 \mathrm{~mm}$ than for the cross section at $X=112 \mathrm{~mm}$.

(2) At each analysis point, the peak value of the spectrum was found at the blade passing frequency and its harmonics in accordance with the speed ratio.

(3) Positive and negative vortex structures were generated alternately in the direction of rotation on both the impeller and turbine.

(4) The vortex structure was subdivided in the direction of rotation at the 2 nd harmonics for a speed ratio of 0.1 . A similar subdivision was also found at speed ratios of 0.4 and 0.7 .

(5) The vorticity distribution of the 2 nd harmonics had a threelayer structure along the axis of rotation for a speed ratio of 0.1 in the direction of flow. However, the subdivided vortex structures merged as the speed ratio increased to 0.4 and 0.7 . It was concluded that the shear flow was caused by the difference in rotational speed between the impeller and turbine, which affects vortex subdivision.

The flow field can be extracted according to the frequency of noise and vibration caused by fluid fluctuations. By analyzing the flow field, the point of interest for the modification of the blade shape can be specified. It can be universally utilized because it is the analysis method that is not dependent on the blade shape or the performance.

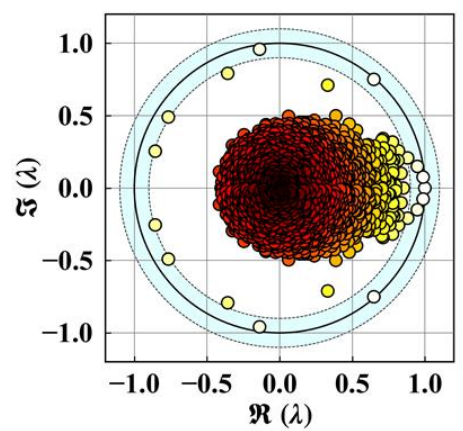

(a)

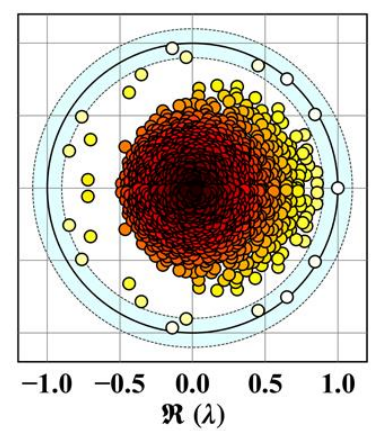

(b)

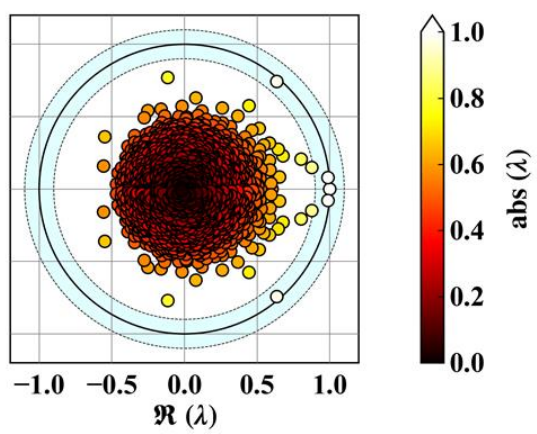

(c)

Fig. 5 Eigenvalue distributions. (a) cross section at $X=91 \mathrm{~mm}$ for speed ratio of 0.1 ,

(b) cross section at $X=91 \mathrm{~mm}$ for speed ratio of 0.7 , (c) cross section at $X=112 \mathrm{~mm}$ for speed ratio of 0.1 .

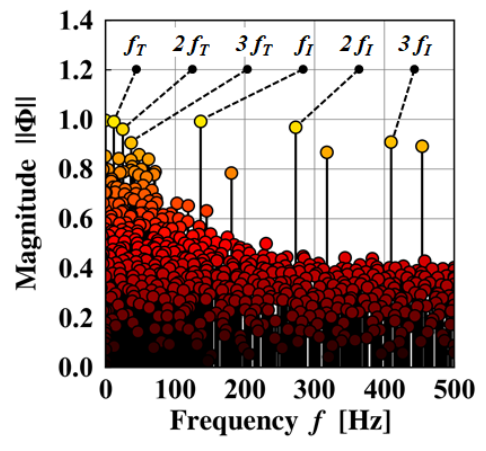

(a) Speed ratio 0.1

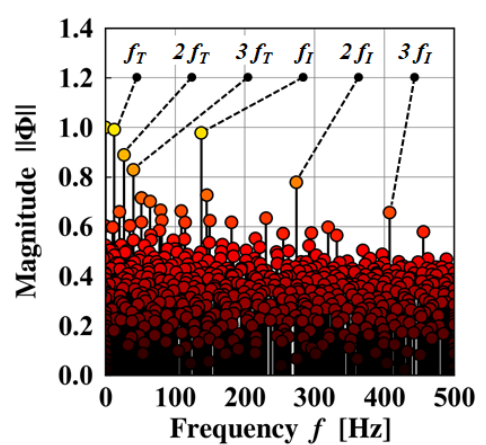

(d) Speed ratio 0.1

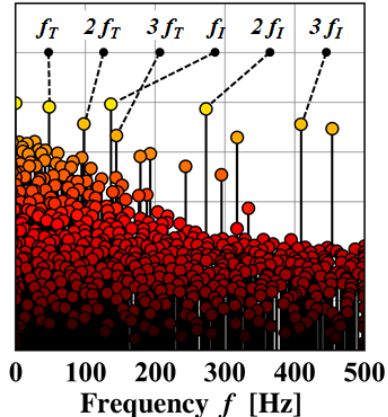

(b) Speed ratio 0.4

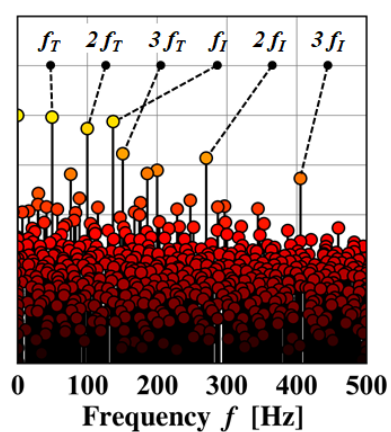

(e) Speed ratio 0.4

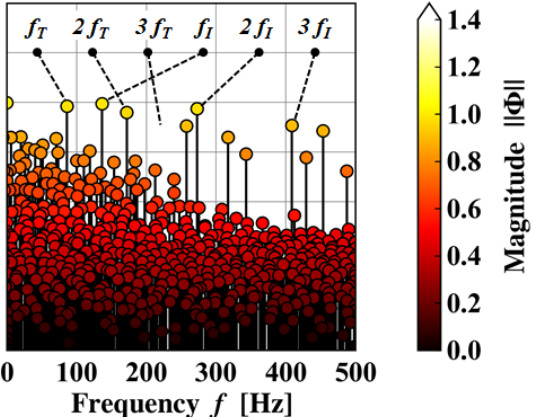

(c) Speed ratio 0.7

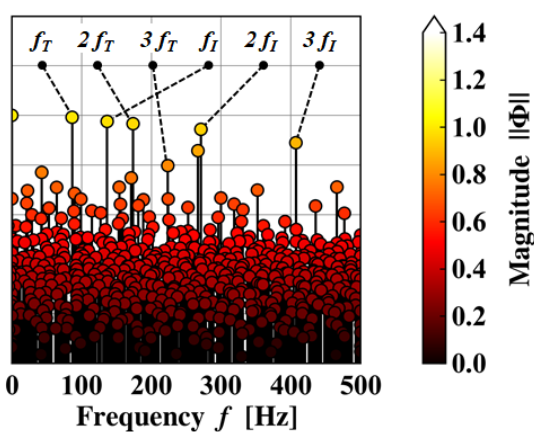

(f) Speed ratio 0.7

Fig. 6 Magnitude of DMD mode. (a) (b) (c) cross section at $X=91 \mathrm{~mm}$, (d) (e) (f) cross section at $X=112 \mathrm{~mm}$. 


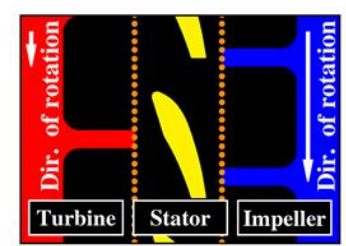

Flow direction
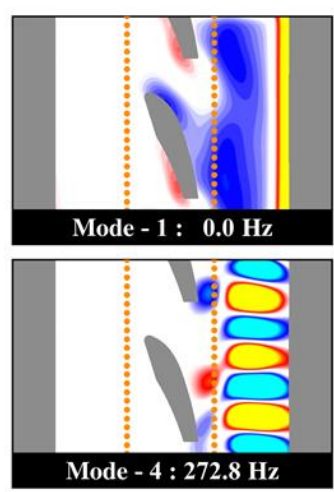
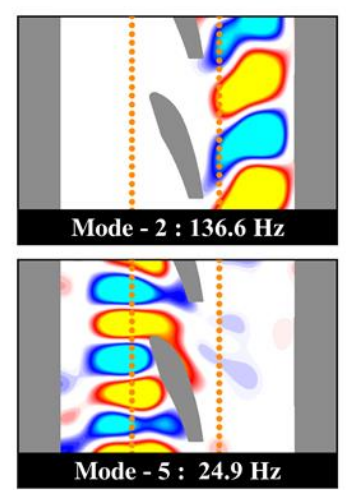
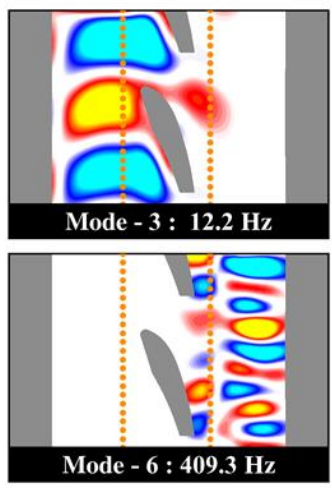

(a) Distributions of vorticity contours
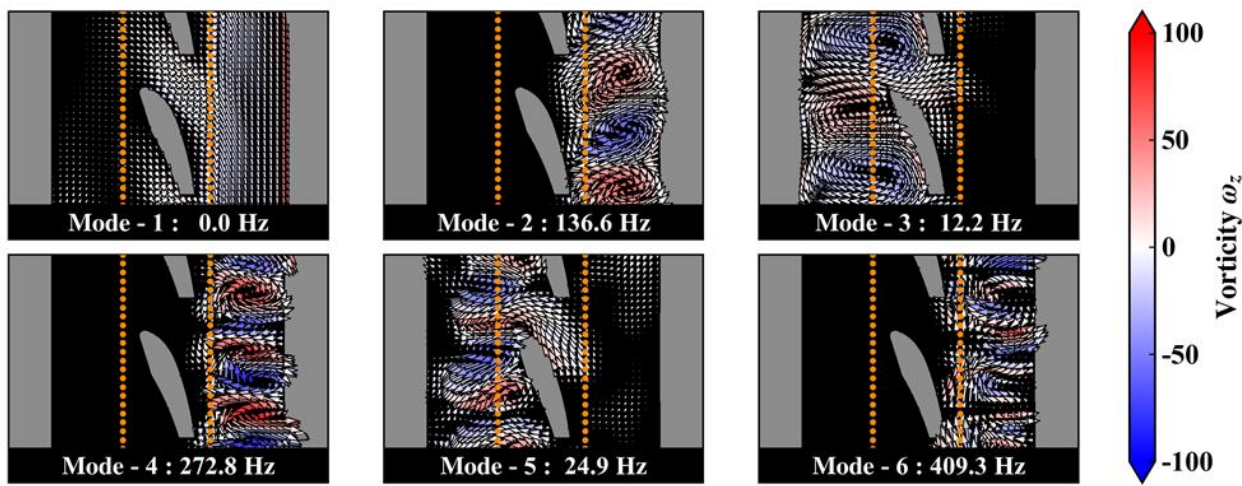

(b) Distributions of velocity vectors

\section{References}

(1) Kobayashi, T., Taniguchi, N., Tasaka, T.:

Three Dimensional Flow Simulation in a Torque Converter: JSAE IPC-8 Technical Paper, No.9531525 (1995)

(2) Tsujita, H., Mizuki, S., Ejiri, E.: Analysis of Flow within Pump Impeller of Torque Converter: ASME Paper, 96-GT404 (1996)

(3) Liu, B., Yan, Q., Wei, W.: Numerical investigations of the flow induced oscillation of a torque converter: Engineering Applications of Computational Fluid Mechanics, Vol.12, No.1, pp.270-281 (2018)

(4) Nandan, A., Sawkar, P, M., Michael, F.: Numerical Investigation of Flow Induced Excitations in a Torque Converter: SAE Technical Paper, No. 2017-01-1115 (2017)

(5) Tasaka, T., Oshima, N., Fujimoto, S., Kishi, Y.: Application of Large Eddy Simulation to a Torque Converter to Predict its Fluid Performance: SAE Technical Paper, No.2017-01-1116 (2017)

(6) Bran, K., Flack, R. D.: Laser Velocimeter Measurements in the Turbine of an Automotive Torque Converter: Part II Unsteady Measurements: ASME Proceedings, No.95-GT293 (1995)

(7) Kawasaki, K., Okada, K., Nishino, K.: Dynamic stereo PIV measurements and 3-D reconstruction of the flow field inside a torque converter model: JSME Fluids Engineering Conference, Vol.2015, 1619 (2015)

(8) Kunisaki, Y., Murata, S., Tanaka, Y., Takeda, T.:
Experimental Analysis for Internal Flow Field of Torque Converter - Application of wavelet analysis to flow field between cascades -: TVSJ, Vol.39, No.5, pp.11-18 (2019) (in Japanese)

(9) P. J. Schmid, J.: Dynamic mode decomposition of numerical and experimental data: Journal of Fluid Mechanics, vol. 656, pp.5-28 (2010)

(10) Ohmichi, Y., Ishida, T., Hashimoto, A.: Numerical Investigation of Transonic Buffet on a Three-Dimensional Wing using Incremental Mode Decomposition: 55th AIAA Aerospace Sciences Meeting, (2017)

(11) Tu, J. H., Rowley, C. W., Luchtenburg, D. M., Brunton, S. L., Kutz, J. N.: On dynamic mode decomposition: theory and applications (2013)

(12) Zhang, W., Wei, M.: Model order reduction using DMD modes and adjoint DMD modes: 8th AIAA Theoretical Fluid Mechanics Conference, (2017)

(13) De Lamotte, A., Delafosse, A., Calvo, S., Delvigne, F., Toye, D.: Investigating the effects of hydrodynamics and mixing on mass transfer through the free-surface in stirred tank bioreactors: Chemical Engineering Science, 172, pp.125-142 (2017)

(14) De Lamotte, A., Delafosse, A., Calvo, S., Toye, D.: Identifying dominant spatial and time characteristics of flow dynamics within free-surface baffled stirred-tanks from CFD simulations: Chemical Engineering Science, 192, pp.128-142 (2018)

(15) The Visualization Society of Japan.: PIV Handbook: Morikita Publishing Co., Ltd., pp.69-75 (2002).

(16) Hart, D. P.: PIV error correction: Experiments in Fluids, Vol.29, Issue 1, pp.13-22 (2000) 
Yasunori Kunisaki et al / International Journal of Automotive Engineering

Vol.11, No.3(2020)

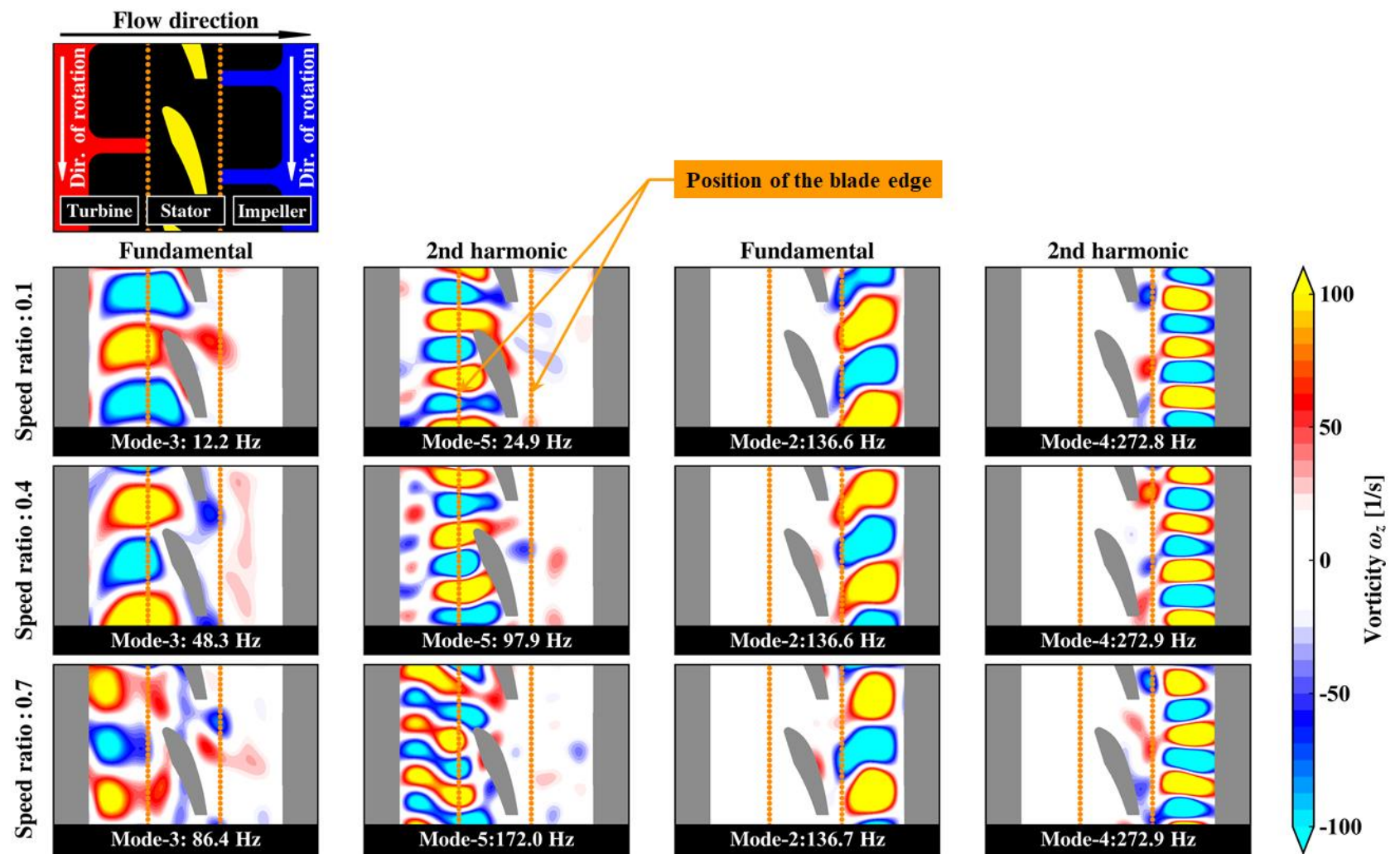

(a) cross section at $X=91 \mathrm{~mm}$
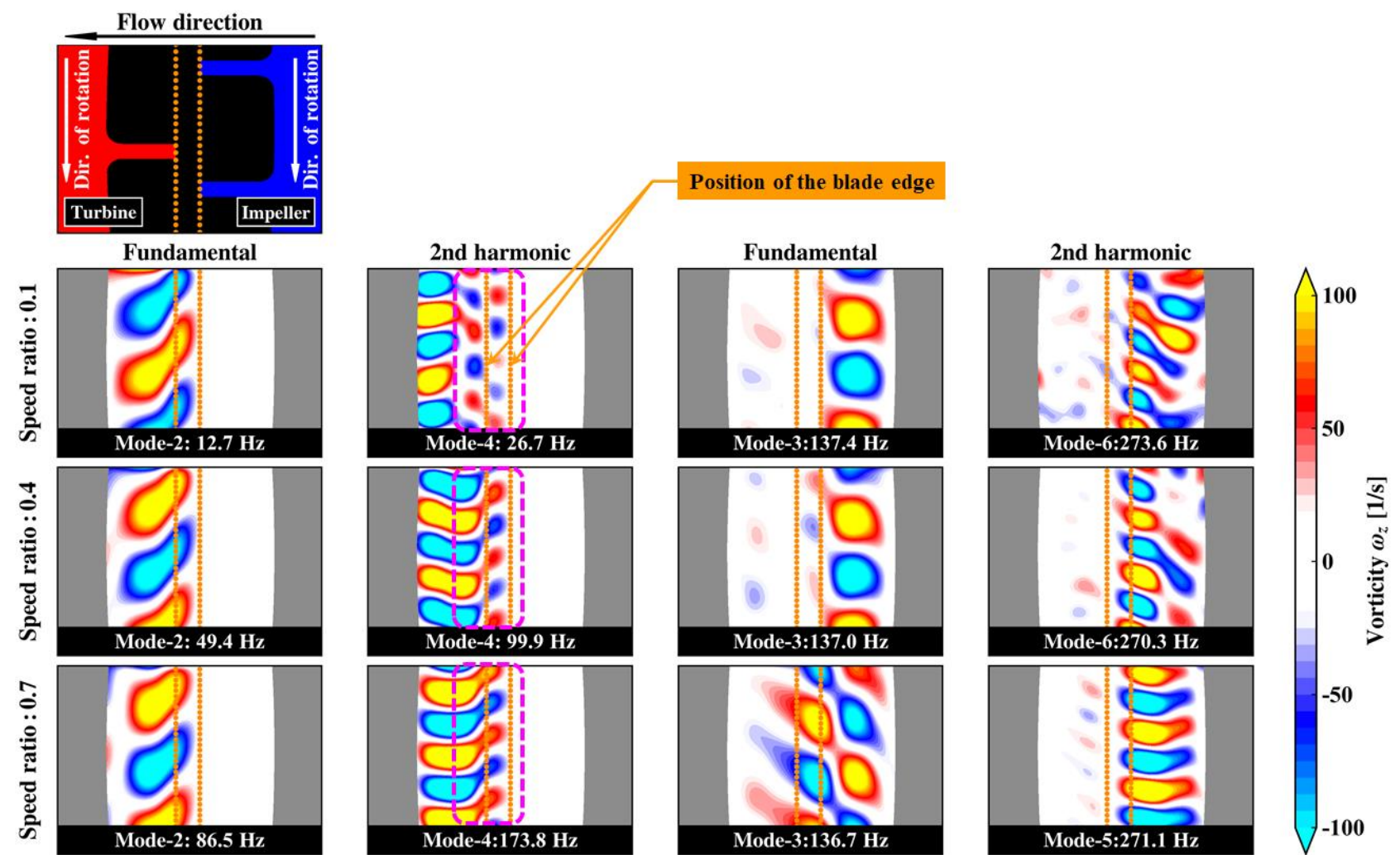

(b) cross section $X=112 \mathrm{~mm}$

Fig. 8 Distributions of vorticity contours for speed ratios of $0.1,0.4$, and 0.7 . 